\title{
Front Matter: Volume 7421
}

, "Front Matter: Volume 7421," Proc. SPIE 7421, The Nature of Light: What are Photons? III, 742101 (2 October 2009); doi: 10.1117/12.848997

SPIE Event: SPIE Optical Engineering + Applications, 2009, San Diego, California, SPIE. United States 


\section{PROCEEDINGS OF SPIE}

\section{The Nature of Light: What are Photons? III}

Chandrasekhar Roychoudhuri

Al F. Kracklauer

Andrei Yu. Khrennikov

Editors

3-4 August 2009

San Diego, California, United States

Sponsored and Published by

SPIE 
The papers included in this volume were part of the technical conference cited on the cover and title page. Papers were selected and subject to review by the editors and conference program committee. Some conference presentations may not be available for publication. The papers published in these proceedings reflect the work and thoughts of the authors and are published herein as submitted. The publisher is not responsible for the validity of the information or for any outcomes resulting from reliance thereon.

Please use the following format to cite material from this book:

Author(s), "Title of Paper," in The Nature of Light: What are Photons? III, edited by Chandrasekhar Roychoudhuri, Al F. Kracklaver, Andrei YU. Khrennikov, Proceedings of SPIE Vol. 7421 (SPIE, Bellingham, WA, 2009) Article CID Number.

ISSN 0277-786X

ISBN 9780819477118

Published by

SPIE

P.O. Box 10, Bellingham, Washington 98227-0010 USA

Telephone +1 3606763290 (Pacific Time) · Fax +1 3606471445

SPIE.org

Copyright (C) 2009, Society of Photo-Optical Instrumentation Engineers

Copying of material in this book for internal or personal use, or for the internal or personal use of specific clients, beyond the fair use provisions granted by the U.S. Copyright Law is authorized by SPIE subject to payment of copying fees. The Transactional Reporting Service base fee for this volume is $\$ 18.00$ per article (or portion thereof), which should be paid directly to the Copyright Clearance Center (CCC), 222 Rosewood Drive, Danvers, MA 01923. Payment may also be made electronically through CCC Online at copyright.com. Other copying for republication, resale, advertising or promotion, or any form of systematic or multiple reproduction of any material in this book is prohibited except with permission in writing from the publisher. The CCC fee code is 0277-786X/09/ $\$ 18.00$.

Printed in the United States of America.

Publication of record for individual papers is online in the SPIE Digital Library.

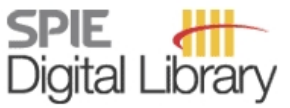

SPIEDigitalLibrary.org

Paper Numbering: Proceedings of SPIE follow an e-First publication model, with papers published first online and then in print and on CD-ROM. Papers are published as they are submitted and meet publication criteria. A unique, consistent, permanent citation identifier (CID) number is assigned to each article at the time of the first publication. Utilization of CIDs allows articles to be fully citable as soon they are published online, and connects the same identifier to all online, print, and electronic versions of the publication. SPIE uses a six-digit CID article numbering system in which:

- The first four digits correspond to the SPIE volume number.

- The last two digits indicate publication order within the volume using a Base 36 numbering system employing both numerals and letters. These two-number sets start with 00, 01, 02, 03, 04, $05,06,07,08,09,0 A, 0 B \ldots 0 Z$, followed by 10-1Z, 20-2Z, etc.

The CID number appears on each page of the manuscript. The complete citation is used on the first page, and an abbreviated version on subsequent pages. Numbers in the index correspond to the last two digits of the six-digit CID number. 


\section{Contents}

vii Conference Committee
ix Introduction

SESSION 1 CLASSICAL AND QUANTUM INTERRELATIONS

742102 Can fluctuations of classical random field produce quantum averages? (Invited Paper) [7421-01]

A. Khrennikov, Växjö Univ. (Sweden)

742103 Spectral properties and nonlinear dynamics of spontaneous emission of a resonance photon by two-level atom trapped in damped nanocavity with a resonance mode [7421-02]

V. Cheltsov, Moscow State Mining Univ. (Russian Federation)

\section{SESSION 2 SUPERPOSITION AND DIVISIBILITY OF PHOTONS I}

742104 A non-quantum mechanical explanation of the single photon double slit experiment (Invited Paper) [7421-03]

K. O. Greulich, Fritz Lipmann Institute (Germany)

742105 Indivisibility of the photon [7421-04]

A. Muthukrishnan, New York Univ. (United States); C. Roychoudhuri, Univ. of Connecticut (United States)

742106 Do light beams cross each other unperturbed? [7421-05]

G. N. Mardari, Rutgers Univ. (United States) and Open Worlds Research (United States);

J. A. Greenwood, Open Worlds Research (United States)

\section{SESSION 3 SUPERPOSITION AND DIVISIBILITY OF PHOTONS II}

742109 The irreducible photon (Invited Paper) [7421-07]

D. L. Andrews, Univ. of East Anglia Norwich (United Kingdom)

7421 OA Exploring divisibility and summability of 'photon' wave packets in nonlinear optical phenomena [7421-08]

N. Prasad, NASA Langley Research Ctr. (United States); C. Roychoudhuri, Univ. of Connecticut (United States)

$7421 \mathrm{OB}$ What can we learn by differentiating between the physical processes behind interference and diffraction phenomena? [7421-09]

Q. Peng, California State Univ., Northridge (United States); A. M. Barootkoob, Consultant (United States); C. Roychoudhuri, Univ. of Connecticut (United States) 
7421 OC Hidden variables: the resonance factor [7421-38]

J. H. J. Brooks, General Resonance LLC (United States)

\section{SESSION 4 RELATIVITY, COSMOLOGY AND ASTROPHYSICS}

7421 OE Demystification of Bell inequality [7421-41]

A. Khrennikov, Växjö Univ. (Sweden)

7421 OF Astrophysical evidence shows no direct interaction between gravitation and electromagnetism in empty vacuum space [7421-12]

E. H. Dowdye, Jr., Pure Classical Physics Research (United States)

$74210 G \quad$ Relativistic transformation of wavelength provides insights into the geometry of photons [7421-14]

M. J. Mobley, Arizona State Univ. (United States)

$7421 \mathrm{OH} \quad$ The natural philosophy of the cosmos (B) [7421-15]

R. C. Storti, Delta Group Engineering, P/L (Australia)

7421 Ol Relativity explained by physical interpretation of coordinates of energy [7421-36]

V. Fernando, Natural Philosophy Alliance (Canada)

\section{SESSION 5 MAXWELL'S EQUATIONS AND ELECTROMAGNETISM}

7421 OM Photon-like solutions of Maxwell's equations in dispersive media (Invited Paper) [7421-17]

J. E. Carroll, J. Beals IV, Univ. of Cambridge (United Kingdom)

$74210 N \quad$ Photon: quantum representation versus field representation seen from a new stand point [7421-18]

E. H. Berloffa, Leopold-Franzens-Univ. Innsbruck - retired (Austria)

$742100 \quad$ Two versions of Maxwell's equations and the nature of light [7421-19]

T. L. Gill, W. W. Zachary, Howard Univ. (United States)

7421 OP Light's two fields [7421-20]

R. Crane, Consultant (United States)

$74210 Q \quad$ Photons as momentum-energy eigenmodes [7421-21]

M. Mazilu, Univ. of St. Andrews (United Kingdom)

\section{SESSION 6 CRITICAL CONCEPTS AND INTERPRETATIONS}

7421 OR Elementary quanta: Are they discrete individual entities? (Invited Paper) [7421-22]

C. Rangacharyulu, Univ. of Saskatchewan (Canada)

7421 OS Another loophole for the Bell inequalities [7421-23]

K. O. Greulich, Fritz Lipmann Institute (Germany) 
7421 OT Hidden variables: the elementary quantum of light [7421-37]

J. H. J. Brooks, General Resonance LLC (United States)

7421 OU Can the quantum theory of light contain the classical electromagnetic theory of light?

[7421-25]

Y. Gan, Hubei Univ. (China)

\section{SESSION 7 CRITICAL CONCEPTS AND INTERPRETATIONS II}

7421 OV EPR-B correlations: a physically tenable local-real model (Invited Paper) [7421-26]

A. F. Kracklauer, Bauhaus Univ. Weimar (Germany)

7421 OW Modified Afshar experiment: calculations [7421-27]

E. V. Flores, Rowan Univ. (United States)

7421 0X Why we need to continue the "What are Photons?" conference: to re-vitalize classical and quantum optics (Keynote Paper) [7421-28]

C. Roychoudhuri, Univ. of Connecticut (United States) and Femto Macro Continuum (United States)

PANEL DISCUSSION: IS INDIVISIBLE SINGLE PHOTON REALLY ESSENTIAL FOR QUANTUM COMMUNICATIONS, COMPUTING AND ENCRYPTION?

7421 OY Is indivisible single photon really essential for quantum communications, computing and encryption? [7421-42]

A. F. Kracklauer, Bauhaus Univ. Weimar (Germany); C. Rangacharyulu, Univ. of Saskatchewan (Canada); C. Roychoudhuri, Univ. of Connecticut (United States) and Femto Macro Continuum (United States); J. H. J. Brooks, General Resonance LLC (United States); J. E. Carroll, Univ. of Cambridge (United Kingdom); A. Khrennikov, Växjö Univ. (Sweden)

\section{POSTER SESSION}

742116 A new approach for solution of the paradox of electron spin [7421-31]

A. Rahmani Nejad, Independent Researcher (Iran, Islamic Republic of); M. A. Olia, Independent Researcher (United States)

742117 A quantum-mathematical model to state single photon (electron) double slit experiment, Fraunhofer and Fresnel diffractions [7421-32]

A. Rahmani Nejad, Independent Researcher (Iran, Islamic Republic of); M. A. Olia, Independent Researcher (United States)

742118 About the nature of light coherence [7421-33]

P. O. Demyanenko, Y. F. Zinkovskij, National Technical Univ. of Ukraine (Ukraine)

742119 A two body photon model [7421-39]

R. T. Dorn, Independent Researcher (United States)

Author Index 
Downloaded From: https://www.spiedigitallibrary.org/conference-proceedings-of-spie on 25 Apr 2023

Terms of Use: https://www.spiedigitallibrary.org/terms-of-use 


\title{
Conference Committee
}

\author{
Conference Chairs
}

Chandrasekhar Roychoudhuri, University of Connecticut (United

States) and Femto Macro Continuum (United States)

Al F. Kracklauer, Bauhaus Universität Weimar (Germany)

Andrei Yu. Khrennikov, Växjö Universitet (Sweden)

Program Committee

Shahriar S. Afshar, Rowan University (United States)

Katherine Creath, Optineering (United States) and College of Optical

Sciences, The University of Arizona (United States)

Benjamin J. Eggleton, The University of Sydney (Australia)

Albrecht Giese, Consultant (Germany)

Karl Otto Greulich, Fritz Lipmann Institute (Germany)

Margaret H. Hawton, Lakehead University (Canada)

Subhash C. Kak, Lovisiana State University (United States)

Akhlesh Lakhtakia, The Pennsylvania State University (United States)

Ashok Muthukrishnan, Texas A\&M University (United States)

John M. Myers, Harvard University (United States)

Narasimha S. Prasad, NASA Langley Research Center (United States)

Vijaya Ramarao, Indian Institute of Technology Bombay (India)

Chary Rangacharyulu, University of Saskatchewan (Canada)

Wolfgang P. Schleich, Universität Ulm (Germany)

Marlan O. Scully, Texas A\&M University (United States) and Princeton University (United States)

Selim M. Shahriar, Northwestern University (United States)

Weilong She, Sun Yat-Sen University (China)

C. S. Unnikrishnan, Tata Institute of Fundamental Research (India)

Ian A. Walmsley, University of Oxford (United Kingdom)

Herbert G. Winful, University of Michigan (United States)

Ewan M. Wright, College of Optical Sciences, The University of Arizona (United States)

\section{Session Chairs}

1 Classical and Quantum Interrelations

Andrei Yu. Khrennikov, Växjö Universitet (Sweden)

Chandrasekhar Roychoudhuri, University of Connecticut

(United States) and Femto Macro Continuum (United States) 
2 Superposition and Divisibility of Photons I

Karl Otto Greulich, Fritz Lipmann Institute (Germany)

Ashok Muthukrishnan, Texas A\&M University (United States)

3 Superposition and Divisibility of Photons II

David L. Andrews, University of East Anglia Norwich (United Kingdom)

Narasimha S. Prasad, NASA Langley Research Center (United States)

$4 \quad$ Relativity, Cosmology and Astrophysics

Michael J. Mobley, Arizona State University (United States)

5 Maxwell's Equations and Electromagnetism

John E. Carroll, University of Cambridge (United Kingdom)

Al F. Kracklauer, Bauhaus Universität Weimar (Germany)

$6 \quad$ Critical Concepts and Interpretations

Al F. Kracklauer, Bauhaus Universität Weimar (Germany)

Chary Rangacharyulu, University of Saskatchewan (Canada)

$7 \quad$ Critical Concepts and Interpretations II

Andrei Yu. Khrennikov, Växjö Universitet (Sweden)

Panel Discussion: Is Indivisible Single Photon Really Essential for Quantum Communications, Computing and Encryption?

Selim M. Shahriar, Northwestern University (United States)

Chandrasekhar Roychoudhuri, University of Connecticut (United States) and Femto Macro Continuum (United States) 


\section{Introduction}

We had a debate on "corpuscular vs. wave" nature of light between Newton and Huygens during early 1700s, which was won by Newton. The next winner was Thomas Young who demonstrated the double-slit experiment during early 1800s to re-establish the wave nature of light. Early 1900 saw a new winner in Einstein who re-introduced the "corpuscular" (particle) concept. Then the quantum physicists appropriated Young's double-slit experiment during 1920s and 1930s to explain the "spooky" "wave-particle duality" of photons without delving into photon-detector interactions that generate the photoelectrons! So, during this new century it is appropriate to re-kindle the debate on the nature of light and the light-matter interaction processes, especially because "spooky" behavior does not appear to be logically self-consistent on the part of nature! It must be the limit of our interpretations and/or our theory. So, we have initiated this conference series encouraging new way of thinking and modeling the nature of light.

This is the $3^{\text {rd }}$ successful biannual conference for this series, which started in 2005, and we have had excellent participation, which clearly underscores the importance of carrying on this conference series. The articles in this volume speak for themselves regarding diversity of new questions that our contributors have raised about the nature of light - from cosmology, general relativity, electromagnetism, quantum and classical statistics, generation and detection of single photons, indivisibility of photons, etc., to the basic interaction processes that give birth to the superposition principle. This year we had a lively panel discussion on the timely topic, "Is indivisible single photon really essential for quantum communications, computing and encryption?" The majority opinion appeared to be negative with a strong dissension from a minority! Active participation by experts from such diverse fields, raising new questions about the lack of complete understanding of the nature of light, is a healthy sign for the progress of physics. After all, EM waves, from Radio waves to gamma rays, have been the most important entity with the ability to provide us with the deeper information about the structures of the material universe, from galaxies to atomic nuclei, without losing their "parental" information they carry, even after they are criss-crossed by innumerable other "beams' and "rays." Thus, we must recognize nature's universal principle, non-interference of EM waves. No other elementary particles provide us with such broad capabilities to explore the inner nature of the material universe.

QM almost recognized this principle of non-interference of light by assigning the properties of non-interacting Bosons to photons, but failed to over-ride the misconception nurtured by classical physics, the notion of "interference of light." Further, enormously successful mathematical human logics behind QM followed 
the century old tradition of developing theories to bring logical congruence among diverse observed results, rather than modeling the invisible interaction processes in detectors that create the data. This enormous gap in our scientific epistemology has been brushed aside as a "measurement problem." Attempts to resolve the issue was repeatedly kept buried under pure mathematical logics, various no-go theorems (specifically Bell's) and an outrageous series of noncausal concepts, to retroactively provide philosophical support to Bohr's claim that quantum mechanics is complete (Einstein lost the debate!). Whereas, as scientists, we are supposed to systematically revise or reconstruct all "successful" theories using new observations, since all human constructed theories are built upon incomplete information about the universe.

The "measurement problem" is not an epistemological problem that we can resolve by using "no-go" theorems. Superposition effect is an active interaction process, not a passive, interaction-free transformation in nature. Superposition principle is always engendered by some active and physical interaction process. Transformations in detectors that we measure are some force guided energy transfer between the detector molecules and the superposed entities. Interaction processes in detectors in EPR-Bell experiments have never been seriously analyzed in the past. The whole problem was reduced to a "loophole" of efficiency of detectors. With respect to tests of Bell inequalities, or realizations of the famous Einstein-Podolsky-Rosen Gedanken experiment, the crucial role of detectors has essentially been taken for granted. It is assumed customarily, that detector output pertains in fact to just what the theory considers the input signal to be. However, this presumption must be verified to be certain that spurious processes (with respect to the considered theory) have not introduced unexpected contributions invalidating the conclusions. Recent work by Adenier, for example, demonstrates that there exist semi-classical processes within detectors (as currently understood) that lead to violations of Bell inequalities without, however, the need to employ the hypotheses that (i) the superposition effect is nonlocal and (ii) photons are indivisible elementary particles. [See citation in Khrennikov paper and Kracklaver's paper in this volume.]

That excited atoms and molecules emit discrete frequencies (classical physics) with discrete packets of energies (quantum mechanics), which we call photons, are well validated. However, whether these photons remain indivisible in spite of their universally accepted diffractive propagation is not as well established. Single "clicks" in "single photon detectors" constitute a current pulse consisting of billions of electrons amplified by the electronic circuit and the biased photo detector, which we assume has been definitely triggered by a single electron. We further assume that it is an "indivisible photon" that triggered the transfer of only a single electron out of the "sea of electrons" of the valence band to the "electron empty sea" of the conduction band. These are lot of assumptions to support the original hypothesis that photons are definitely "indivisible" [See papers in this volume by Muthukrishnan, Prasad, and Peng]. 
Recall that photons are non-interacting Bosons, implying that light beams do not rearrange their energy distributions to create "interference fringes" in the absence of material detectors. We could have saved our energy for almost a century by not considering "single photon interference" as a debatable issue, had we explicitly recognized nature's principle of non-interference of EM waves. EM fields do not sum by themselves. QM has more realities built into its successful formalism than we have been willing to explore. The resultant dark-bright fringes in superposition experiments are consistent with the QM prescription - the energy transfer to the detecting molecules is the square modulus of the sum of all the conjoint dipolar stimulations, which are the complex amplitudes of dipole stimulations; not simply sum of the EM fields. We have evolved thinking that we "see" light, even though it is only our cerebral interpretation of the signal sent by the detecting retinal molecular transformations. So, we have been stuck in thinking that a dark fringe means non-arrival of photons or absence of light energy; whereas it is the absence of any transformation of the detecting molecules due to simultaneous out-of-phase stimulations by the superposed fields. Superposition effect is local since the detecting molecules must simultaneously experience all the superposed fields.

For our $4^{\text {th }}$ biannual conference in 2011, we want our current and future participants to appreciate that our conference platform will remain as broad as the frequency range of EM waves, from Radio waves to gamma-rays! We strongly encourage authors to submit papers that attempt to imagine, visualize and explain the real physical processes behind the generation, propagation and detection of light; thereby energize the debate on whether light consists of indivisible quanta, divisible wave packets, or something new. All submitted theoretical and experimental papers should deal with actual or feasible experiments to prove their points in deference to pure mathematically formalistic papers primarily on quantum philosophy, pure quantum logic or "Gedanken" experiments.

The rest of this introduction is a modified version of the 2009-Call for papers to underscore our promise to keep the conference platform widely open, as well as to give readers insight into questions that were posed to participants at the outset of the 2009 conference. May we request that our current authors and other readers feel free to copy this introduction and circulate among potential new authors to submit papers for the 2011 conference?

\section{Generic Physics questions:}

\section{Principle of Non-Interference of Light (NIL)}

Can a single photon create "interference" effects all by itself? Are photons indivisible quanta, or divisible classical wave packets? 


\section{Causality of time-frequency Fourier theorem}

Should we continue to use Fourier's mathematical time-frequency theorem as a de-facto principle of physics, as if superposed light beams interfere with each other, even though they constitute non-interacting Bosons, or non-interfering classical wave packets?

\section{Relativity \& Reality of cosmic medium as sustainer of light}

Why do we continue to ignore the field properties of the space as unreal when Maxwell's wave equation and quantum field theory of light are based upon such fields? How can light travel through real empty space with the same possible highest velocity irrespective of the velocity of the emitters?

\section{Semi-Classical Optics questions (quantized atoms and classical wave packets):}

\section{Diffraction vs. interference}

Classical mathematical formulation for both these phenomena are fundamentally same, we sum the component EM waves, whether they are secondary wavelets produced due to perturbation by apertures or they are superposed well-formed beams. But, if light beams constitute photons and photons are non-interacting Bosons, then classical physics has been wrong for centuries to assume "interference" of light. Is classical physics correct?

\section{Fourier transform spectroscopy}

Why does Michelson's Fourier transform spectroscopy work, even though different frequencies produce heterodyne signals, while Michelson derived his relation by assuming that different frequencies do not interfere?

\section{Mode-lock laser pulse}

If light beams of different frequencies do not interfere, why do we claim that mode-locked laser pulses are produced by superposition of periodic longitudinal modes? Why do the multi-mode $\mathrm{CW}$ gas lasers produce steady $\mathrm{CW}$ intensity instead of random pulses even though the "temporal coherence" of each mode in over millisecond?

\section{Elliptically polarized beam}

If orthogonally polarized light cannot interfere to produce fringes, how can they interfere to produce elliptically polarized beam? If the amplitude of the electric vector in an elliptically polarized light oscillates in its value, would not the energy of the beam (square of the amplitude) oscillate?

\section{Dispersion: Fourier frequencies of a pulse and pulse broadening}

If the response time of atoms and molecules to incident light beam is in the domain of femto seconds or shorter, then how do material media figure out how to respond to Fourier frequencies of pico or nano second pulses that barely touched the material facet? What are the physical processes by which the molecules of a "dispersive" medium figures out the presence of Fourier frequencies due to short pulses?

\section{Slow and fast light}

Is "superluminal" velocity of light a physical reality or limitations of our mathematical model that use Fourier frequencies? 


\section{Coherence theory}

If light beams constitute non-interacting Bosons, then why do we present coherence of light beams as field-field correlation instead of as correlation of simultaneous dipole stimulations of the detecting molecules simultaneously induced by multiple superposed fields?

\section{Coherence and decoherence}

If diffractive propagation of all classical beams show enhancement in coherence (van Cittert-Zernike theorem), how can we reconcile decoherence of light beams as they evolve through propagation?

\section{Classical spectroscopy}

The principle of conservation of energy in the real world dictates that all light signals constitutes finite pulses. The, why we do not derive classical spectroscopic formula by directly propagating the carrier frequency of pulses, while taking care of their time-finite durations? Can a passive grating really decompose a pulse into its component Fourier frequencies and then separate them out? Then why do we need nonlinear optical media t5o generate new frequencies?

\section{Resolution limit of classical spectroscopy}

Classical physics accepts the fundamental limit of spectrometric resolving power limit as $\delta v \delta t \geq 1$ for pulse of width $\delta t$. This is only a corollary of the time-frequency Fourier theorem. Can this mathematical corollary of the time-frequency Fourier theorem be a principle of nature when the parent theorem is not?

\section{Quantum Optics questions (both atoms and light are quantized):}

\section{Electrons are discrete but "photons" may not be}

If electrons are stable and quantized elementary particles, and their binding energies are quantized in detectors, how can we be so decisive that discrete number of photoelectron emission proves that light is also discrete? Does QM bar all quantum devices from absorbing the necessary quantum of energy from multiple stimulators?

\section{Single photon generation}

Since emitting and detecting molecules are nanometer in size and the wavelengths of light are two to three orders of magnitude larger, can we trust the hypothesis of single photon generation or detection unless the emitter/detector is an isolated single atom?

\section{"Photon interferes only with itself"}

Can we accept this proposal by Dirac as final when we know that heterodyne spectrometry can be carried out by superimposing beams of light on a fast detector from a star some 13-billion light-years far and an Earth-based laser?

\section{Photons do not arrive at dark fringe locations}

Should we accept the explanation that superposition fringes (whether spatial or temporal) are produced due to preferential arrival of photons as the final resolution of Dirac's proposal? All transformations happen in nature through interactions and energy exchange. How can a single photon make itself appear or disappear without any physical interaction? 


\section{Photon as a Fourier monochromatic mode of the vacuum}

Is it logically congruent for us to accept the definition for a photon as a Fourier monochromatic mode of the "vacuum"? A Fourier monochromatic mode is a non-causal proposition since it mathematically exists over all space and time!

\section{Non-locality}

If the fringes of superposition due to light beams become manifest only in the presence of nanometer size detecting molecules, present within the physical volume of superposition of the beams, how can interference phenomenon be non-local?

\section{Validity of Bell's theorem}

If the fringes of superposition due to light beams become manifest only in the presence of detecting molecules, should not the Bell's theorem be re-derived in terms of summation of the simultaneous dipole stimulations by the light beams?

\section{$a \& a+$ as repackaged $A \& B$ coefficients}

Since "creation" and "annihilation" of photons in reality are always carried out by material dipoles, do a \& a+ really represent Einstein's "AB" coefficients?

\section{Quantum computation by single photons}

Is it possible to track the same single photon through the multiple stages of generation, propagation, manipulation and detection processes for quantum computation, encryption and communication, when the light-matter interaction is always statistical?

\section{Nano photonics and Plasmonic photonics}

If quantum, in contrast to bulk, material properties start dominating in nano photonic materials, why do we not need propagating quantized EM fields in such devices? Why classical diffractive propagation using Maxwell's wave equation yields perfectly valid results?

\section{Optical trapping and Vortex optics}

Can the experimental advancements in these fields help us discern between "indivisible photon vs. classical wave packet?"

\section{Bose-Einstein Condensates}

Since photons are Bosons, can we leverage the advancements in BEC physics to understand the nature of light, photons vs. wave packets, any better?

\section{Particle physics}

Since photons and elementary particles can give birth to each other, can the study of the structure of light lead to better understanding of the structure of particles? 